\title{
Individual flexor tendon identification within the carpal tunnel:A semi-automated analysis method for serial cross-section magnetic resonance images
}

This article was published in the following Dove Press journal:

Orthopedic Research and Reviews

3 December 2009

Number of times this article has been viewed

\author{
Nicole M Kunze' \\ Jessica E Goetz ${ }^{2}$ \\ Daniel R Thedens ${ }^{3}$ \\ Thomas E Baer ${ }^{2}$ \\ Ericka A Lawler ${ }^{2}$ \\ Thomas D Brown² \\ 'Department of Biomedical \\ Engineering, ${ }^{2}$ Department of \\ Orthopaedics and Rehabilitation, \\ ${ }^{3}$ Department of Radiology, University \\ of lowa, lowa City, IA, USA
}

\begin{abstract}
Carpal tunnel syndrome is commonly viewed as resulting from chronic mechanical insult of the median nerve by adjacent anatomical structures. Both the median nerve and its surrounding soft tissue structures are well visualized on magnetic resonance (MR) images of the wrist and hand. Addressing nerve damage from impingement of flexor digitorum tendons co-occupying the tunnel is attractive, but to date has been restricted by a lack of means for making individual identifications of the respective tendons. In this image analysis work, we have developed a region-growing method to positively identify each individual digital flexor tendon within the carpal tunnel by tracking it from a more distal MR section where the respective tendon identities are unambiguous. Illustratively, the new method was applied to MRI scans from four different subjects in a variety of hand poses. Conventional shape measures yielded less discriminatory information than did evaluations of individual tendon location and arrangement. This new method of rapid identification of individual tendons will facilitate analysis of tendon/nerve interactions within the tunnel, thereby providing better information about mechanical insult of the median nerve.
\end{abstract}

Keywords: carpal tunnel syndrome, magnetic resonance imaging, region growing, digital flexor tendons

\section{Introduction}

Carpal tunnel syndrome (CTS) is the most commonly encountered peripheral compressive neuropathy. The paresthesia, numbness, and muscle weakness that characterize this condition are caused by mechanical insult of the median nerve as it courses through the osseoligamentous carpal tunnel, which is co-occupied by nine digital flexor tendons. ${ }^{1}$ While extensive research has focused on ergonomic and work-place factors associated with the development of CTS, relatively little is known about the actual mechanism of direct mechanical insult to the median nerve.

Information about soft tissue interactions within the carpal tunnel can be obtained using magnetic resonance imaging (MRI). Magnetic resonance imaging offers exquisite resolution of the soft tissues of the tunnel (the median nerve, the digital flexor tendons, and the transverse carpal ligament), and therefore has been used to quantify and document characteristics of both the normal and pathologic conditions. ${ }^{2-6}$ In T1-weighted MR images of the carpal tunnel, the median nerve typically appears as an intermediate signal intensity structure with a stippled texture (Figure 1 ). ${ }^{7}$ By contrast, both the transverse carpal ligament and the flexor tendons have low MR signal intensity and appear black, with individual tendons being delineated by contrast with the higher signal intensity of the surrounding tendon sheaths. ${ }^{8}$
Correspondence: Jessica E Goetz Orthopaedic Biomechanics Lab, 2180 Westlawn Building, lowa City, IA 52242-I 100, USA

$\mathrm{Tel}+\mathrm{I} 3193844275$

Fax +I 3193357530

Email jessica-goetz@uiowa.edu 


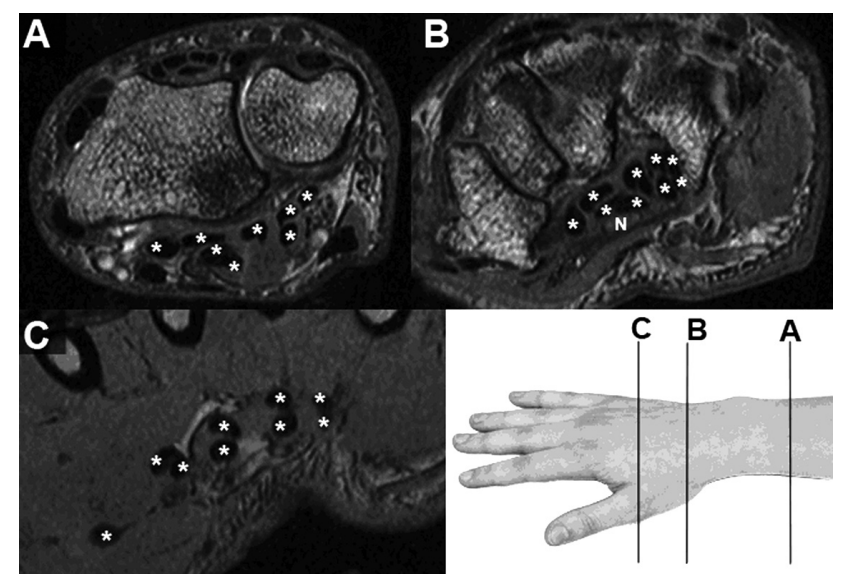

Figure I Magnetic resonance images, with flexor tendons identified $(*)$ within B) and on either side of (A and $\mathbf{C}$ ) the carpal tunnel. Only in the distal image from the hand $\mathbf{C}$ ), where the tendons align in pairs going to each finger (one to the thumb) and in two layers (deep and superficial), is unambiguous tendon identification possible. The median nerve $(\mathbf{N}$, in panel $\mathbf{B})$ has stippled image texture of intermediate signal intensity.

A subset of MRI-based studies related to CTS has focused on the size and shape of selected structures in the carpal tunnel, attempting to identify pathological changes in these characteristics associated with CTS. In several early structure/shape studies using low field strength MRI scanners, various anatomic features of the carpal tunnel were cataloged at different locations in the hand for both normal volunteers and for cadavers. ${ }^{2,6}$ From such studies, the typical cross-sectional area of the median nerve in the normal carpal tunnel has been found to be between 7 and $8 \mathrm{~mm}^{2}$, with the nerve noted to be slightly flattened. ${ }^{6}$ It has also been found that there is a slight volar bowing of the transverse carpal ligament in normal wrists. ${ }^{2}$ In these types of structure/shape studies, four main features have been identified that are commonly associated with carpal tunnel syndrome: enlargement of the median nerve, flattening of the median nerve, bulging of the transverse carpal ligament, and increased signal intensity of the median nerve on T2-weighted images. ${ }^{3,9}$ Occasional attempts have been made to stage the severity of CTS based on progressive changes of these key criteria. ${ }^{10}$

In such studies, the ratio of the volume of the carpal tunnel contents to the total carpal tunnel volume has also been a parameter of interest, as a greater ratio would indicate either a smaller tunnel or enlarged tunnel tissues. Both factors intuitively would tend to increase crowding, and thus pressure on the median nerve. ${ }^{11}$ Measurements of the volume of the carpal tunnel occupied by soft tissues are usually expressed as a ratio of carpal tunnel contents to carpal tunnel volume, with values in the range of $50 \%-60 \%$ being typical in axial sections through the narrowest part of the tunnel. ${ }^{12,13}$ Comparisons of this ratio made between CTS patients and normals have been used to quantify abnormally increased soft tissue in the carpal tunnel, and therefore the potential for increased pressure on the median nerve from adjacent soft tissue structures. ${ }^{13}$ Overall carpal tunnel size, specifically cross-sectional area visible on axial MRI sections, appears to be slightly smaller (although not statistically significantly so) in CTS patients than in normal subjects. ${ }^{14}$ As a practical matter, however, such investigations have found very similar volumetric filling ratios in CTS patients and in normal subjects. ${ }^{13}$

Despite observations of MRI-apparent abnormalities in nerve signal intensity, nerve size, nerve flattening ratio, and changes in overall tunnel size and contents ratio, MRI has been found to be relatively nonspecific and insensitive for CTS. ${ }^{15,16}$ Changes in signal intensity associated with "magic-angle" effects (increased signal intensity as well-ordered tendon collagen fibers approach a $55^{\circ}$ orientation relative to the direction of the main magnetic field) can artificially indicate CTS pathology. ${ }^{17}$ Diagnosis of CTS based on changes in nerve MR signal intensity can also be confounded by the fact that nerve signal intensity may either increase or decrease relative to normal, depending on the severity of CTS symptoms. ${ }^{10}$ Therefore, the primary diagnostic modalities employed to identify CTS have been physical examination and electrodiagnostic nerve conduction tests, with MRI examination reserved for cases where the diagnosis is either unclear ${ }^{9,18}$ or confounded by additional pathology. ${ }^{9,16}$ MRI presumably could play a greater diagnostic role if the pertinent anatomical abnormalities evident on MR images were more definitively linked to CTS pathology.

Because CTS is a result of mechanical insult to the median nerve, MRI studies of the carpal tunnel anatomy and function have understandably focused primarily on abnormalities of the nerve, and somewhat secondarily on abnormalities of the transverse carpal ligament. By contrast, relatively few attempts have been made to characterize abnormalities of the digital flexor tendons. One preliminary attempt has been made to track the tendons through the carpal tunnel by connecting centroids of the flexor tendons in sequential MRI sections. ${ }^{19}$ However, those data were used only to determine the radius of curvature of the idealized pulley associated with the wrapping of the tendons around adjacent anatomic structures during flexion and extension, ${ }^{11}$ rather than to evaluate the tendons' potential interactions with the median nerve and with each other.

One impediment to systematically studying tendon-nerve interaction has been that it is difficult to know which tendon 
is which once inside the carpal tunnel. One study made an effort to determine the anatomic location of the median nerve relative to specific tendons, but due to the laborious nature of attempting to manually identify specific tendons within the carpal tunnel, structure arrangements were investigated only at rather coarse $(6 \mathrm{~mm})$ intervals, and only for a selected few subjects. ${ }^{20}$ The challenge of identifying specific tendons results from the identities of individual tendons being ambiguous due to inconsistency of stacking arrangements both between subjects and in different wrist postures. Adding to the ambiguity of tendon identification is the high mobility of these structures during movement of the hand. Therefore, definitive tendon identification must be made outside the carpal tunnel and propagated back into the tendons of the tunnel. The purpose of this work was to develop and implement a method for rapidly and definitively identifying the specific individual digital flexor tendons within the carpal tunnel, for the purpose of including flexor tendon abnormalities in MRI evaluations of carpal tunnel syndrome.

\section{Methods}

Three-dimensional MR images of the wrists of four subjects (two male, two female) were acquired using a wrist or a lower-extremity transmit/receive coil, depending on the subjects' wrist size. One of the subjects had clinically-diagnosed CTS, whereas the other three subjects had no history of wrist pain and were considered to have normal wrists. Scans were performed on a Siemens TIM Trio 3T (Siemens Medical, Malvern, PA, USA) scanner using either a 90-second, or a threeminute, dual echo steady state (DESS) pulse sequence (TR/TE $=13.0 / 4.3 \mathrm{~ms}$ ), with water excitation to suppress signal from fat tissue. The acquired resolution for the three-minute DESS sequence was $0.15 \mathrm{~mm} \times 0.15 \mathrm{~mm} \times 0.8 \mathrm{~mm}$ voxels over an $8 \mathrm{~cm} \times 6 \mathrm{~cm} \times 7.7 \mathrm{~cm}$ field of view. The acquired resolution for the reduced, ninety-second DESS sequence was $0.2 \mathrm{~mm} \times$ $0.2 \mathrm{~mm} \times 1.0 \mathrm{~mm}$ voxels over an $8 \mathrm{~cm} \times 6 \mathrm{~cm} \times 7.5 \mathrm{~cm}$ field of view. Wrist positions were varied for each subject, and ranged from $35^{\circ}$ of flexion to $35^{\circ}$ of extension, either with or without a hand loading fixture. A total of 15 scan volumes from the four subjects were utilized for analysis.

For each scan, MR sections containing the proximal edge of the pisiform and the distal hook of the hamate were used to define the proximal and distal ends of the carpal tunnel, respectively. Using a set of purpose-written Matlab (The Mathworks, Natick, MA, USA) programs, the median nerve, the nine digital flexor tendons, and the carpal tunnel boundary were manually segmented on a set of axial images spanning the length (typically 25 to $30 \mathrm{~mm}$ ) of the carpal tunnel. These programs sequentially opened all images spanning the carpal tunnel, and automatically presented those images to the user, along with a series of push-button menus which directed the user to segment the carpal tunnel structures. Tendon boundaries were determined by finding and tracing the boundary where the greatest contrast appeared between the (dark) tendon and (lighter surrounding) tissue. The median nerve appeared as a textured, enclosed fascicular region within the carpal tunnel. Thus, the median nerve boundary was determined by tracing between the light gray textured region and the typical band of encircling low-signalintensity. The carpal tunnel boundary was determined by finding the low signal intensity transverse carpal ligament on the volar side of the tunnel and the dark cortical shell of the carpal bones on the dorsal side of the tunnel (Figure 1). On average, approximately $30 \mathrm{MRI}$ sections through the carpal tunnel were segmented per wrist.

Following segmentation within the carpal tunnel per se, each tendon was individually identified using a regiongrowing technique that tracked the tendon identity into the carpal tunnel, beginning from a distal location where the identity was unambiguous (Figure 1). ${ }^{6}$ A distal MRI section from within the hand, in which each tendon could be easily distinguished, was used as the starting image for the region-growing technique. Each of the nine tendons on the starting image was manually identified and outlined in the same order: thumb, superficial index, deep index, superficial long, deep long, superficial fourth, deep fourth, superficial little, and deep little, respectively (Figure 2). This tracing order ensured each tendon received the same identifier in

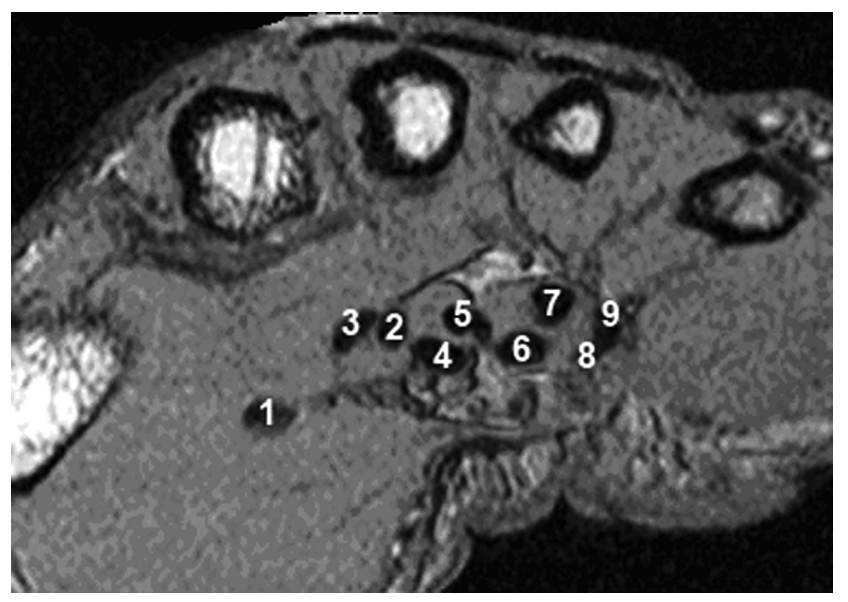

Figure $\mathbf{2}$ In order to ensure that each tendon receives the same numeric label (shown above) in any analyzed scan, tendons are always outlined in the same order at the beginning of the tracking.

Notes: I, thumb; 2, superficial index; 3 , deep index; 4, superficial long; 5, deep long; 6, superficial fourth; 7, deep fourth, 8 , superficial little; 9 , deep little. 
every scan, and the initial outline surrounding the structure provided an individual size threshold for that structure, thereby preventing the necessity of establishing a set threshold within the algorithm. The algorithm for tracking individual tendon identities is summarized schematically in Figure 3. The area within each tendon boundary was calculated and used as a growth limitation threshold to ensure that one tendon region did not grow so large as to merge into another. The centroid of the initially-outlined tendon cross-sectional area was used as the center of a $3 \times 3$ seed region from which to grow the tendon region on the next-proximal MR image in the series. Each tendon region was grown using a four-connected neighborhood comparison scheme, with a candidate pixel being added to the provisional tendon cross-section if its intensity $\left(I_{\text {candidate }}\right)$ was in the appropriate grayscale range for the overall tendon cross-section $\left(I_{\text {mean_of_seed }}\right)$ :

$$
\left|I_{\text {Candidate }}-I_{\text {mean_of_seed }}\right| \leq 0.8 * I_{\text {mean_of_seed }}
$$

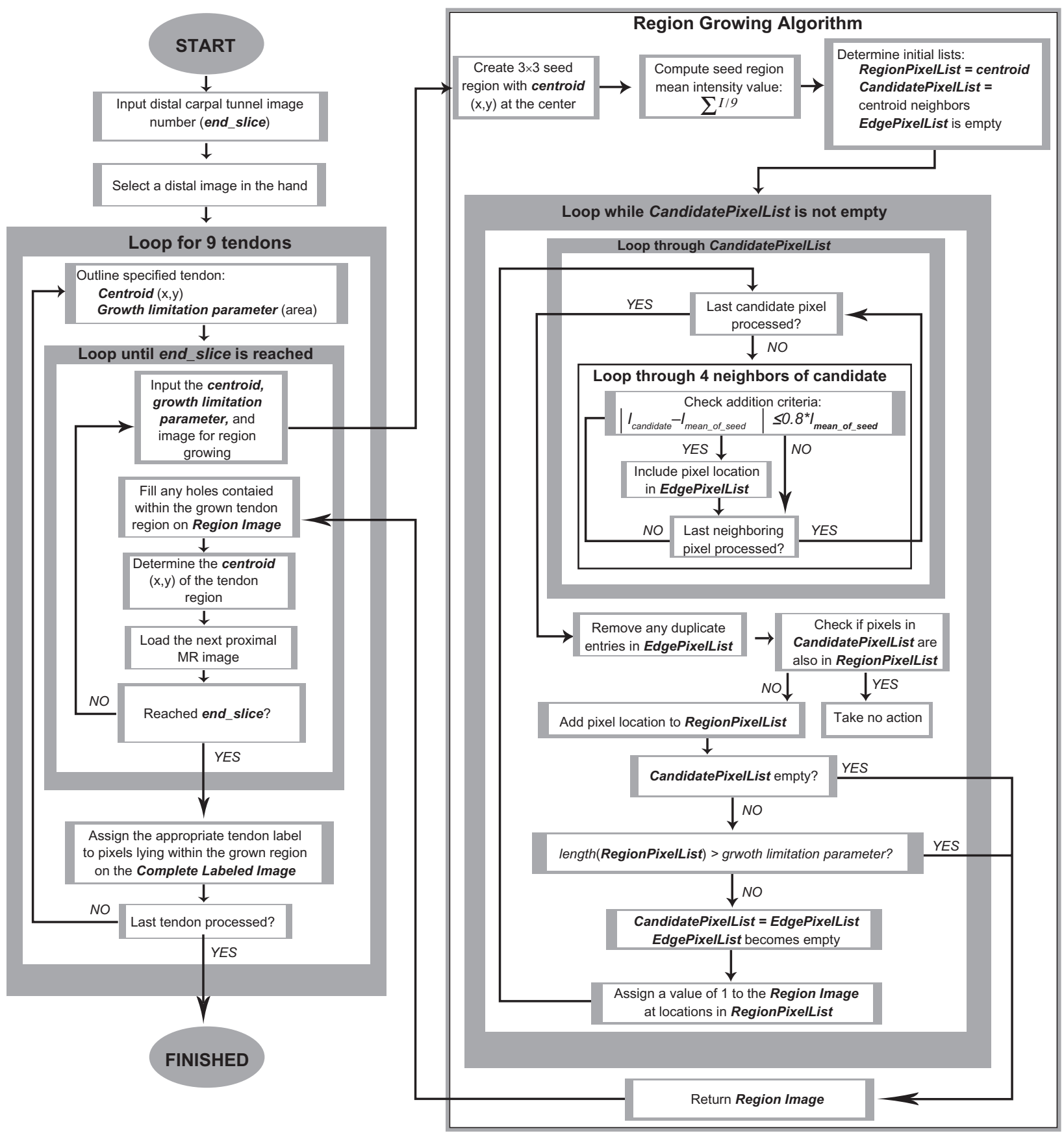

Figure 3 Flowchart detailing the steps of the tendon identification algorithm. 
In a given MRI section, a tendon region continued to grow until either there were no remaining candidate boundary pixels, or until its area had reached the area growth limitation threshold, determined as the area within the initially traced boundary. The centroid of the resulting grown region was then used to define the $3 \times 3$ seed region from which to grow the tendon region on the next-proximal image, and so forth. The tendon tracking process was complete once the sectionby-section region growing for all nine tendons had propagated to the image delimiting the distal margin of the carpal tunnel (Figure 4). The result of this region-growing and tracking process was a labeled image, corresponding to the distal-most carpal tunnel image, containing the identities of each grown tendon region. Each grown tendon region was identified with a number 1 through 9, corresponding to each of the digital flexor tendons (thumb $=1$, superficial tendons $=$ even digits, and deep tendons $=$ odd digits). To most reliably obtain the labeled image, region-growing was required in MRI sections taken at intervals of at most $2 \mathrm{~mm}$ between the starting distal hand location and the distal margin of the carpal tunnel.

The labeled image resulting from the region-growing and tracking process was then used to conclusively identify each of the tendons in all sections that had been segmented within the carpal tunnel. Tendon identities were automatically propagated from the labeled image through the segmentations by determining in which region on the label image the centroids of the unknown tendons on the distal end of the carpal tunnel segmentations were located. The segmented regions in the carpal tunnel were given the same identifier as the corresponding region in the labeled image. The identified segmentations were used to create 3D models of the carpal tunnel illustrating the median nerve, the nine flexor tendons, and the tunnel boundary. This was done by enclosing each identified region within an isosurface generated using Matlab. The same numeric identifier was assigned to the same tendon in any image series processed with this method, thereby allowing for assessment of intra-subject tendon movement with wrist motion, and inter-subject comparisons of tendon location, movement, and stacking arrangement.

Making use of the known tendon identities, traditional CTS-related size and shape measurements were illustratively tabulated for all carpal tunnel structures (nerve, tunnel, and each tendon) in every axial section of each wrist scan. These data were extracted from the manually segmented axial MRI sections using code developed in Matlab that implemented program-specific functions which operate on binary images. Size measures included cross-sectional area, perimeter, major axis length, and minor axis length. Cross-sectional area was
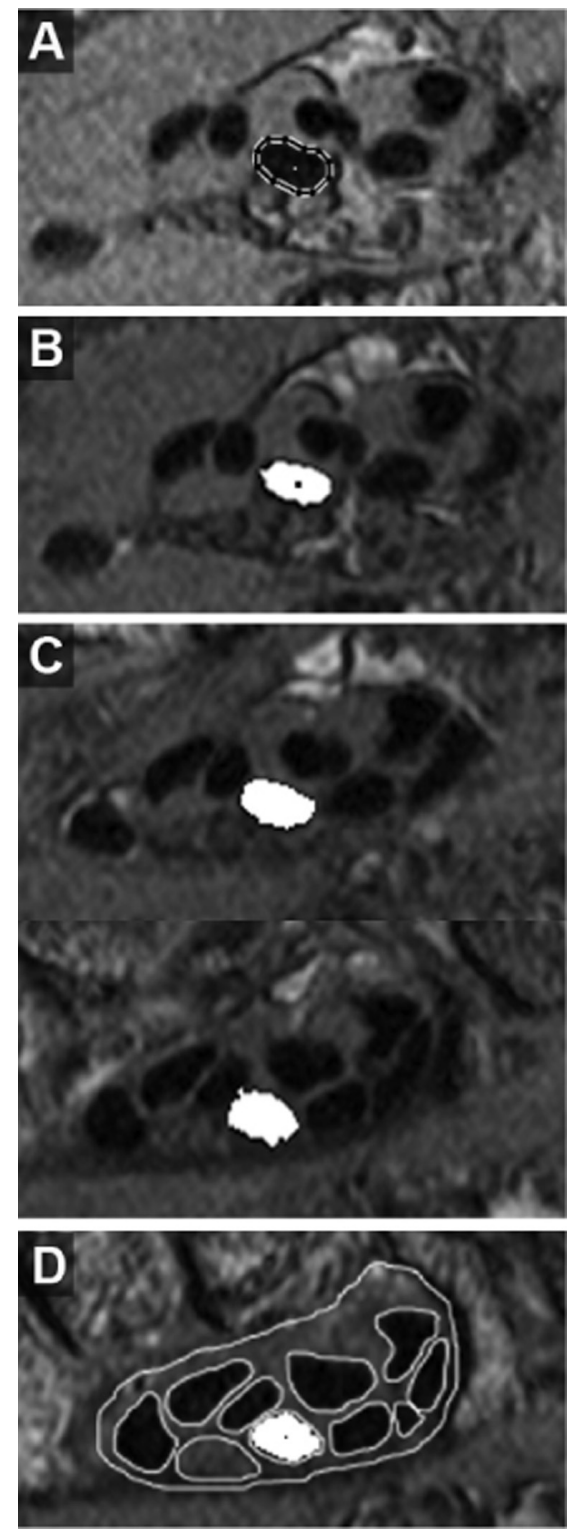

Figure 4 Progressive automatic tracking of the 3rd digit superficial tendon, beginning from the starting image.The centroid of the outlined tendon boundary $(\mathbf{A})$ is used to establish the seed region (B) from which the next tendon region is grown. Additional cross-sections of this tendon at six-section increments, moving proximally toward the distal end of the carpal tunnel are shown in $\mathbf{C}$. Tracking continues until the image of the distal carpal tunnel is reached, which coincides with the distal-most segmentations (D).

found by obtaining the number of pixels within each segmented region and converting to square millimeters, by multiplying pixel counts by the in-plane voxel dimensions. The perimeter was found by calculating the distance between adjoining pairs of eight-connected pixels around the border of each segmented region, and major and minor axis lengths were calculated to be those of an ellipse fit to the boundary pixels of each structure. A carpal tunnel contents ratio was also calculated as the sum of the cross-sectional areas of the tendons and nerve divided by the cross-sectional area of the carpal tunnel. The primary 
shape measure calculated from these size data was a flattening ratio, defined as the minor axis length divided by major axis length. ${ }^{14,21,22}$ Because the identities of the structures in the tunnel were known from the tendon tracking algorithm, the size/shape results were automatically catalogued, by structure, in Microsoft Excel spreadsheets (Microsoft Corp., Redmond, WA, USA) for inter- and intrasubject comparisons.

An additional MRI evaluation metric was developed to utilize the tendon identity information in a manner that may be a potential indicator of CTS. The specific individual tendons neighboring the median nerve were determined for every axial section through the carpal tunnel in all 15 scans. First, the boundaries of the tendons, nerve, and carpal tunnel were determined relative to the centroid of the nerve in each axial section. These structure boundary coordinates were then converted to polar coordinates, and the closest adjacent structure to the nerve boundary (tendon or transverse carpal ligament) was determined in $5^{\circ}$ increments around the nerve. A given structure was considered to be adjacent to the nerve if it was within $1 \mathrm{~mm}$ of the boundary of the nerve. From these adjacency data, a percentage of the nerve boundary adjacent to tendons, the amount of nerve boundary shared with each individual flexor tendon, and the approximate angle from the centroid of the nerve to where each tendon was located were measured. The location angle was additionally converted to an anatomic direction relative to the in-plane MR image.

\section{Results}

In all instances, the tendon-tracking algorithm was able to successfully propagate tendon identity information from the unambiguous distal MRI section (in the hand) to the distal boundary of the carpal tunnel segmentation data set. 3D models of the identity-labeled segmentations allowed for direct visual comparison of the soft tissue structures in the carpal tunnels of the four different subjects in a wide variety of hand poses (Figures 5 and 6).

Carpal tunnel contents ratios in a section through the hook of the hamate calculated from the identified segmentations ranged from $47 \%-58 \%$, which was within the range typically seen through the narrowest part of the tunnel. This was an indication that the manual segmentations were realistic and comparable to previous work. Conventional size and shape measures were computed from these segmentations for each identified structure in every axial section spanning the carpal tunnel, thereby allowing changes in these characteristics to be quantified for specific individual structures. One such comparison made was the change in size and shape measures for each structure in the tunnel according to longitudinal location within the tunnel. The results for change in individual tendon cross sectional area with longitudinal location within the tunnel are shown in Table 1. A second comparison that was made was the variation between individual tendon, median nerve, and whole tunnel flattening ratios with differences in hand posture and loading (Table 2).

Upon comparing the tabulated size and shape data with the $3 \mathrm{D}$ models of each wrist scan, it became apparent that trends in the conventional size and shape measures from each axial section provided relatively little indication of the more dramatic changes of the tunnel contents that were evident in the $3 \mathrm{D}$ models as a result of different wrist postures. The relatively nondiscriminatory nature of conventional size and shape measures is evident in Table 1 (percent change in
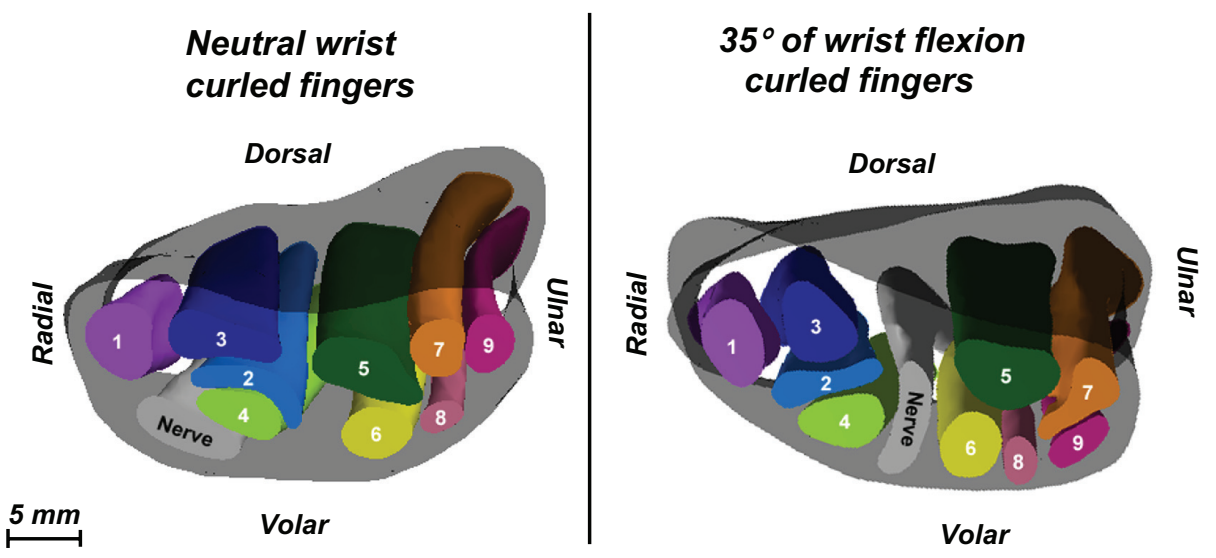

Figure 53 D isosurface models of Subject I's wrist in neutral (left) and in $35^{\circ}$ of flexion (right). This is a proximal-to-distal view. In the respective sections through the narrowest part of the tunnel (at the hook of the hamate), the flattening ratios of the nerve are very similar, despite the very different nerve positions within the tunnel. Notes: I, thumb; 2, superficial index; 3, deep index; 4, superficial long; 5, deep long; 6, superficial fourth; 7, deep fourth, 8, superficial little; 9, deep little. 


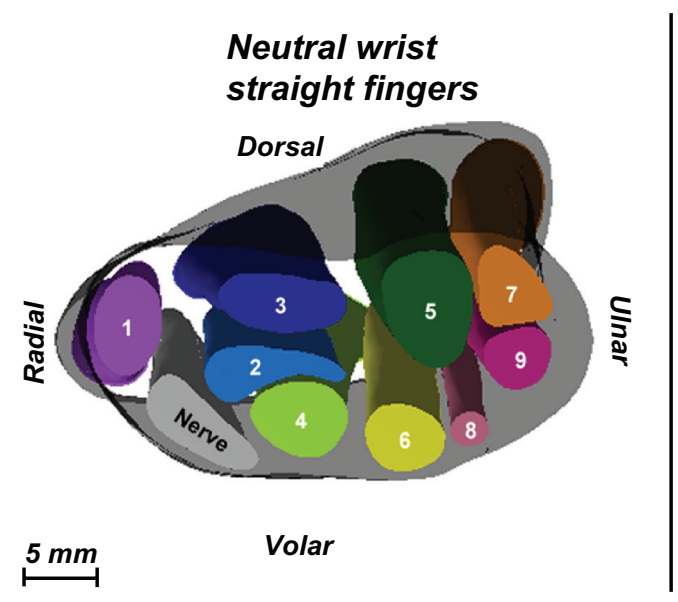

\section{$35^{\circ}$ of wrist flexion curled fingers}

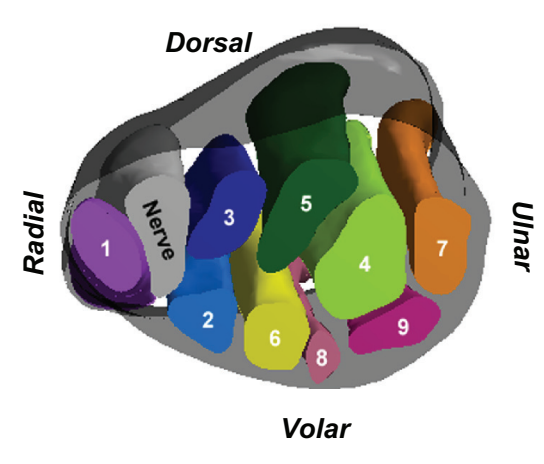

Figure $63 \mathrm{D}$ isosurface models of Subject 2's wrist in neutral (left) and in $35^{\circ}$ of flexion (right). This again is a proximal-to-distal view. The flattening ratio of the nerve decreased very slightly between neutral and flexion, indicating minimal changes to the shape of the nerve. However, in the 3D models, a dramatic change in position is evident for the nerve, the superficial long tendon (2), and the tendons of the 4 th and 5 th digits (6-9).

structure cross-sectional area from proximal to distal) and Table 2 (the structure flattening ratios calculated in a single section through the hook of the hamate). No specific trends in changes of structure cross sectional area as a function of longitudinal position were seen with changes in hand posture (Table 1). Nerve flattening ratios from an axial section at the hook of the hamate for Subject 1 with either a neutral or a flexed wrist were only marginally different, with values of
0.46 and 0.55 respectively. However, upon examination of the 3D models of this subject's wrist in the two different positions (Figure 5), the nerve appeared to have frankly extruded between the index and long tendons as the wrist moved from neutral to $35^{\circ}$ of flexion. The minimal difference between nerve flattening ratios associated with the wrist motion performed by this subject gave no indication of the dramatic difference in nerve position evident when

Table I Size analysis: percent change in structure cross-sectional areas at the distal-most axial section through the carpal tunnel relative to the most proximal axial tunnel section. These values, arising from the size measures of the structures on each axial section, show little indication of the dramatic changes apparent in the 3D models

\begin{tabular}{|c|c|c|c|c|c|c|c|c|c|c|c|c|c|c|}
\hline \multirow[b]{2}{*}{ SUBJECT } & \multirow[b]{2}{*}{ WRIST } & \multirow[b]{2}{*}{ FINGER } & \multirow[b]{2}{*}{ ACTIVITY } & \multirow[b]{2}{*}{ NERVE } & \multirow[b]{2}{*}{ TUNNEL } & \multirow[b]{2}{*}{ THUMB } & \multicolumn{2}{|c|}{ INDEX } & \multicolumn{2}{|c|}{ LONG } & \multicolumn{2}{|c|}{ FOURTH } & \multicolumn{2}{|c|}{ LITTLE } \\
\hline & & & & & & & Sup & Deep & Sup & Deep & Sup & Deep & Sup & Deep \\
\hline I & $\mathrm{N}$ & $S$ & - & 15 & -11 & -17 & -17 & 9 & 0 & 37 & 14 & 10 & -25 & 4 \\
\hline I & $\mathrm{N}$ & C & G & -8 & -6 & -2 & II & -10 & 8 & -43 & -23 & 34 & $-4 I$ & -21 \\
\hline I & $\mathrm{N}$ & C & - & -20 & -10 & 2 & -6 & -15 & 7 & -23 & -11 & 33 & -32 & 5 \\
\hline I & $35 \mathrm{~F}$ & C & - & 22 & -4 & -23 & 7 & -2 & 4 & -25 & 21 & 54 & -33 & -1 \\
\hline I & $35 \mathrm{~F}$ & C & G & -13 & -5 & -36 & -37 & -19 & 41 & -43 & -13 & 8 & -15 & 13 \\
\hline 2 & $\mathrm{~N}$ & $S$ & - & -15 & -7 & -15 & 4 & 12 & 23 & 7 & -9 & 50 & -18 & -18 \\
\hline 2 & $35 \mathrm{~F}$ & C & - & 2 & -17 & -1 & 0 & 7 & -30 & -27 & -41 & -8 & -45 & -37 \\
\hline 3 & $35 \mathrm{E}$ & $S$ & FP & 46 & 1 & 20 & 17 & 9 & 15 & 32 & -20 & 70 & -53 & -1 \\
\hline 3 & $35 \mathrm{E}$ & $S$ & - & -30 & -15 & 17 & -13 & -33 & 3 & 7 & 26 & 40 & -21 & -36 \\
\hline 3 & $\mathrm{~N}$ & S & $\mathrm{FP}$ & 15 & -13 & -20 & 15 & -22 & 27 & -8 & 5 & 2 & -32 & -29 \\
\hline 3 & $\mathrm{~N}$ & $S$ & - & 13 & -11 & 14 & -2 & -27 & 3 & -8 & 2 & -2 & -57 & 23 \\
\hline 4 & $35 \mathrm{E}$ & C & - & 57 & -3 & -1 & -7 & -14 & 1 & -33 & -9 & 95 & -16 & 87 \\
\hline 4 & $\mathrm{~N}$ & $S$ & - & I & 2 & 34 & -17 & 9 & 12 & -12 & -21 & 21 & -19 & -4 \\
\hline 4 & $\mathrm{~N}$ & C & - & 73 & -4 & 25 & 2 & 22 & 6 & -21 & 10 & 30 & -32 & 14 \\
\hline 4 & $35 \mathrm{~F}$ & C & - & 71 & 11 & -17 & 42 & 8 & 2 & -11 & I & 3 & -28 & -15 \\
\hline
\end{tabular}

Abbreviations: Wrists were either in $35^{\circ}$ of extension $(35 \mathrm{E})$, neutral $(\mathrm{N})$, or $35^{\circ}$ of flexion $(35 \mathrm{~F})$, and fingers were either straight $(\mathrm{S})$ or curled $(\mathrm{C})$ loosely around a largediameter rest-bar. Activities were either none (-), squeeze grip (G), or whole-hand volar press (FP). 
Table 2 Shape analysis: flattening ratios of each carpal tunnel structure calculated for an axial section located at the hook of the hamate. Flattening ratio was defined as the minor axis divided by the major axis. Again, these values give little indication of the dramatic changes apparent in the 3D models

\begin{tabular}{|c|c|c|c|c|c|c|c|c|c|c|c|c|c|c|}
\hline \multirow[b]{2}{*}{ SUBJECT } & \multirow[b]{2}{*}{ WRIST } & \multirow[b]{2}{*}{ FINGER } & \multirow[b]{2}{*}{ ACTIVITY } & \multirow[b]{2}{*}{ NERVE } & \multirow[b]{2}{*}{ TUNNEL } & \multirow[b]{2}{*}{ THUMB } & \multicolumn{2}{|c|}{ INDEX } & \multicolumn{2}{|c|}{ LONG } & \multicolumn{2}{|c|}{ FOURTH } & \multicolumn{2}{|c|}{ LITTLE } \\
\hline & & & & & & & Sup & Deep & Sup & Deep & Sup & Deep & Sup & Deep \\
\hline 1 & $\mathrm{~N}$ & $S$ & - & 0.52 & 0.42 & 0.62 & 0.44 & 0.43 & 0.60 & 0.79 & 0.71 & 0.50 & 0.61 & 0.32 \\
\hline I & $\mathrm{N}$ & C & G & 0.42 & 0.44 & 0.61 & 0.42 & 0.47 & 0.67 & 0.87 & 0.53 & 0.57 & 0.39 & 0.36 \\
\hline I & $\mathrm{N}$ & C & - & 0.46 & 0.43 & 0.64 & 0.43 & 0.46 & 0.61 & 0.65 & 0.63 & 0.57 & 0.74 & 0.32 \\
\hline I & $35 \mathrm{~F}$ & C & - & 0.55 & 0.44 & 0.60 & 0.40 & 0.76 & 0.64 & 0.65 & 0.54 & 0.65 & 0.57 & 0.48 \\
\hline 1 & $35 \mathrm{~F}$ & C & G & 0.58 & 0.43 & 0.73 & 0.67 & 0.71 & 0.58 & 0.76 & 0.65 & 0.37 & 0.59 & 0.42 \\
\hline 2 & $\mathrm{~N}$ & $S$ & - & 0.59 & 0.49 & 0.54 & 0.46 & 0.43 & 0.80 & 0.66 & 0.86 & 0.68 & 0.72 & 0.58 \\
\hline 2 & $35 \mathrm{~F}$ & C & - & 0.54 & 0.58 & 0.63 & 0.61 & 0.50 & 0.51 & 0.42 & 0.57 & 0.38 & 0.77 & 0.35 \\
\hline 3 & $35 \mathrm{E}$ & $S$ & FP & 0.92 & 0.49 & 0.53 & 0.73 & 0.55 & 0.79 & 0.36 & 0.96 & 0.63 & 0.62 & 0.47 \\
\hline 3 & $35 \mathrm{E}$ & $S$ & - & 0.37 & 0.48 & 0.57 & 0.42 & 0.50 & 0.63 & 0.80 & 0.73 & 0.59 & 0.62 & 0.33 \\
\hline 3 & $\mathrm{~N}$ & $S$ & FP & 0.40 & 0.45 & 0.68 & 0.38 & 0.39 & 0.65 & 0.84 & 0.66 & 0.87 & 0.64 & 0.93 \\
\hline 3 & $\mathrm{~N}$ & $S$ & - & 0.30 & 0.44 & 0.57 & 0.47 & 0.54 & 0.57 & 0.74 & 0.86 & 0.58 & 0.86 & 0.36 \\
\hline 4 & $35 \mathrm{E}$ & $C$ & - & 0.54 & 0.47 & 0.63 & 0.53 & 0.43 & 0.61 & 0.55 & 0.62 & 0.51 & 0.81 & 0.31 \\
\hline 4 & $\mathrm{~N}$ & $S$ & - & 0.61 & 0.52 & 0.62 & 0.51 & 0.45 & 0.82 & 0.82 & 0.76 & 0.49 & 0.56 & 0.33 \\
\hline 4 & $\mathrm{~N}$ & C & - & 0.62 & 0.48 & 0.57 & 0.42 & 0.37 & 0.75 & 0.83 & 0.60 & 0.43 & 0.88 & 0.44 \\
\hline 4 & $35 \mathrm{~F}$ & C & - & 0.68 & 0.55 & 0.81 & 0.49 & 0.69 & 0.78 & 0.82 & 0.95 & 0.56 & 0.75 & 0.54 \\
\hline
\end{tabular}

Abbreviations: Wrists were either in $35^{\circ}$ of extension $(35 \mathrm{E})$, neutral $(\mathrm{N})$, or $35^{\circ}$ of flexion $(35 \mathrm{~F})$, and fingers were either straight $(\mathrm{S})$ or curled $(\mathrm{C})$ loosely around a largediameter rest-bar. Activities were either none (-), squeeze grip (G), or whole-hand volar press (FP).

observing the 3D models. Moreover, the nerve presumably underwent pronounced squeezing by those two tendons during that transition.

The flattening ratios of the nerve and carpal tunnel for Subject 2, with wrist positions of neutral and flexed, reflected only a slight change in shape, but again there were large changes in the positions of tendons observed in the 3D models (Figure 6). The flattening ratios of the nerve were 0.59 and 0.54 and of the tunnel were 0.49 and 0.58 for the wrist in neutral and in $35^{\circ}$ of flexion, respectively. However, the nerve in the $3 \mathrm{D}$ models appeared to extrude between the thumb and index tendons as the wrist moved from neutral to flexion, and the nearby superficial long and the fourth and fifth digit tendons adopted a very different in-plane arrangement. These position and configuration changes, while again dramatic and highly suggestive of appreciable tendon-nerve interaction during the transient, were not reflected in the conventional shape measures obtained from axial sections.

To more appropriately register these position-shift phenomena, the identities of the structures adjacent to the nerve, the amount of adjacency, and the approximate location of each adjacent tendon relative to the nerve were extracted for each axial section of every MRI scan. For illustrative purposes, these data are shown for a section selected from each scan containing the hook of the hamate (Figure 7). Tendons missing from the data table in Figure 7 are those which were not adjacent to the nerve in any of the evaluated hamate sections. The first four columns identify the subject as well as the angle of the wrist, configuration of the fingers, and the loading activity (if one was used). The next column identifies the total percent of the nerve boundary that is adjacent (defined as $1 \mathrm{~mm}$ or less proximity) to tendons and the carpal tunnel boundary. A higher percentage of adjacency indicated that more of the nerve perimeter was contacting or closely neighbored by a carpal tunnel structure. Upon comparing overall percentages of nerve boundary adjacent to another structure, Subject 1's nerve showed appreciably more variation in the amount of adjacency to other structures during different hand poses, ranging from $17 \%$ to $58 \%$.

Table 3 shows the approximate location of each structure adjacent to the nerve in the plane of the MR section. By comparing the locations of each structure between different wrist positions, it was readily apparent when the nerve had changed its relative position within the carpal tunnel. For example, considering Subject 1 with curled fingers moving from neutral to wrist flexion, the position change of the nerve that was visible in Figure 5 was also apparent when comparing relative locations of the structures in Table 3. This was 

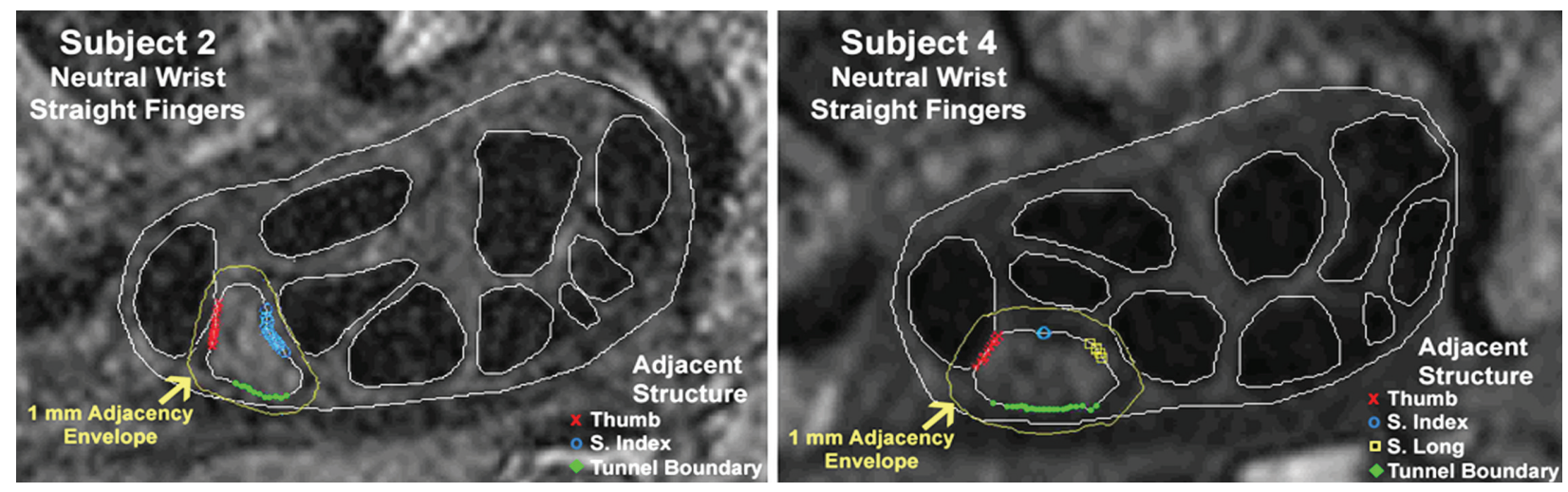

\begin{tabular}{|c|c|c|c|c|c|c|c|c|c|c|c|}
\hline SUBJECT & WRIST & FINGER & ACTIVITY & $\%$ ADJACENCY & TUNNEL & THUMB & $\begin{array}{l}\text { INDEX } \\
\text { Sup }\end{array}$ & $\begin{array}{c}\text { INDEX } \\
\text { Deep }\end{array}$ & $\begin{array}{l}\text { LONG } \\
\text { Sup }\end{array}$ & $\begin{array}{l}\text { LONG } \\
\text { Deep }\end{array}$ & $\begin{array}{c}\text { FOURTH } \\
\text { Sup }\end{array}$ \\
\hline 1 & $\mathrm{~N}$ & $\mathrm{~s}$ & -- & $58 \%$ & 130 & 25 & 25 & 0 & 30 & 0 & 0 \\
\hline 1 & $\mathrm{~N}$ & $C$ & $G$ & $56 \%$ & 140 & 0 & 0 & 0 & 60 & 0 & 0 \\
\hline 1 & $\mathrm{~N}$ & C & -- & $46 \%$ & 125 & 0 & 0 & 0 & 40 & 0 & 0 \\
\hline 1 & $35 \mathrm{~F}$ & C & -- & $26 \%$ & 0 & 0 & 5 & 0 & 60 & 0 & 30 \\
\hline 1 & $35 \mathrm{~F}$ & C & G & $17 \%$ & 0 & 0 & 0 & 0 & 50 & 10 & 0 \\
\hline 2 & $\mathrm{~N}$ & $\mathrm{~s}$ & -- & $58 \%$ & 65 & 65 & 80 & 0 & 0 & 0 & 0 \\
\hline 2 & $35 \mathrm{~F}$ & C & -- & $53 \%$ & 95 & 55 & 0 & 40 & 0 & 0 & 0 \\
\hline 3 & $35 \mathrm{E}$ & $\mathrm{s}$ & FP & $32 \%$ & 70 & 0 & 5 & 0 & 40 & 0 & 0 \\
\hline 3 & $35 \mathrm{E}$ & $S$ & -- & $60 \%$ & 130 & 0 & 45 & 0 & 40 & 0 & 0 \\
\hline 3 & $\mathrm{~N}$ & $\mathrm{~s}$ & FP & $26 \%$ & 25 & 0 & 0 & 0 & 70 & 0 & 0 \\
\hline 3 & $\mathrm{~N}$ & $\mathrm{~s}$ & -- & $54 \%$ & 100 & 0 & 70 & 0 & 25 & 0 & 0 \\
\hline 4 & $35 \mathrm{E}$ & C & -- & $40 \%$ & 125 & 0 & 0 & 0 & 20 & 0 & 0 \\
\hline 4 & $N$ & $S$ & -- & $54 \%$ & 115 & 45 & 10 & 0 & 25 & 0 & 0 \\
\hline 4 & $\mathrm{~N}$ & C & -- & $43 \%$ & 130 & 0 & 0 & 0 & 25 & 0 & 0 \\
\hline 4 & $35 \mathrm{~F}$ & C & -- & $56 \%$ & 145 & 35 & 20 & 0 & 0 & 0 & 0 \\
\hline
\end{tabular}

Figure 7 Nerve adjacency analysis: adjacency of the nerve to the surrounding structures at the hook of the hamate. The locations of adjacency are plotted on two segmented MRI sections (top).

Notes: Data associated with the images are marked in the table with a $*$. In the table (bottom), \% adjacency is the percentage of the nerve boundary within I mm of a tunnel structure, and the numerical value associated with each tendon or the tunnel boundary is the number of degrees along the nerve boundary lying within I mm of that respective structure.

Abbreviations: Wrists were either in $35^{\circ}$ of extension $(35 \mathrm{E})$, neutral $(\mathrm{N})$, or $35^{\circ}$ of flexion $(35 \mathrm{~F})$, and fingers were either straight $(\mathrm{S})$ or curled $(\mathrm{C})$ loosely around a largediameter rest-bar. Activities were either none (-), squeeze grip (G), or whole-hand volar press (FP).

especially evident in the different location of the superficial long tendon relative to the nerve. In the neutral wrist position (Subject 1, N, C) the superficial long tendon was located ulnarly, while in the flexed wrist position (Subject 1, 35F, C) the superficial long tendon was volar. Thus, this tendon appeared on a different side of the nerve in the flexed wrist as compared to the neutral wrist. In this same pair of scans, the tunnel boundary, which was volarly adjacent to the nerve in the neutral position, was no longer within $1 \mathrm{~mm}$ of the nerve in the flexed position. Similarly, the superficial index and superficial fourth tendons, which did not engage the nerve in the neutral wrist position, became adjacent to the nerve in the flexed position (radially and volar-ulnarly, respectively). Furthermore, the extrusion of Subject 2's nerve through the tendons with wrist flexion that was evident in the $3 \mathrm{D}$ tunnel models (Figure 6) was also apparent in the location data in Table 3. For example, the approximate location of the thumb tendon was dorsal-radial to the nerve in the neutral position, and radial to the nerve in the flexed position. The deep index tendon moved into adjacency and the superficial index tendon moved out of adjacency, as the wrist moved from neutral to flexion. Furthermore, the location of the nerve's adjacency to the carpal tunnel boundary changed to the opposite side of the nerve, reflecting the nerve now moving into contact with the dorsal aspect of the tunnel rather than the volar. Based upon such observations, these novel measures of adjacency hold attraction for meaningfully describing abnormalities and variations of relative positions of carpal tunnel soft tissue structures, information not available from conventional shape measures. 
Table 3 Structure location analysis: approximate location of each structure adjacent to the nerve

\begin{tabular}{|c|c|c|c|c|c|c|c|c|c|c|}
\hline & & & & & & INDEX & INDEX & LONG & LONG & FOURTH \\
\hline SUBJECT & WRIST & FINGER & ACTIVITY & TUNNEL & THUMB & Sup & Deep & Sup & Deep & Sup \\
\hline I & $\mathrm{N}$ & $S$ & - & $\mathrm{V}$ & $R$ & DU & - & $U$ & - & - \\
\hline I & $\mathrm{N}$ & C & G & V & - & - & - & DU & - & - \\
\hline I & $\mathrm{N}$ & C & - & V & - & - & - & $U$ & - & - \\
\hline I & $35 \mathrm{~F}$ & C & - & - & - & $\mathrm{R}$ & - & $\mathrm{V}$ & - & VU \\
\hline 1 & $35 \mathrm{~F}$ & C & G & - & - & - & - & V & $U$ & - \\
\hline 2 & $\mathrm{~N}$ & S & - & V & DR & $U$ & - & - & - & - \\
\hline 2 & $35 \mathrm{~F}$ & C & - & $D$ & $\mathrm{R}$ & - & VU & - & - & - \\
\hline 3 & $35 \mathrm{E}$ & $S$ & FP & V & - & DR & - & $\mathrm{D}$ & - & - \\
\hline 3 & $35 \mathrm{E}$ & $S$ & - & V & - & $D$ & - & DU & - & - \\
\hline 3 & $\mathrm{~N}$ & $S$ & FP & V & - & - & - & $\mathrm{D}$ & - & - \\
\hline 3 & $\mathrm{~N}$ & $S$ & - & V & - & D & - & DU & - & - \\
\hline 4 & $35 \mathrm{E}$ & C & - & V & - & - & - & DU & - & - \\
\hline 4 & $\mathrm{~N}$ & $S$ & - & V & DR & D & - & DU & - & - \\
\hline 4 & $\mathrm{~N}$ & C & - & $\mathrm{V}$ & - & - & - & DU & - & - \\
\hline 4 & $35 \mathrm{~F}$ & C & - & VR & $D$ & DU & - & - & - & - \\
\hline
\end{tabular}

Abbreviations: Wrists were either in $35^{\circ}$ of extension (35E), neutral $(\mathrm{N})$, or $35^{\circ}$ of flexion $(35 \mathrm{~F})$, and fingers were either straight $(\mathrm{S})$ or curled $(\mathrm{C})$ loosely around a largediameter rest-bar. Activities were either none (-), squeeze grip $(G)$, or whole-hand volar press (FP). Locations are specified as radial (R), ulnar (U), dorsal (D), volar (V), or a combination of two directions.

\section{Discussion}

This study reports a new method for automatically tracking the individual identities of the digital flexor tendons into the carpal tunnel in sets of segmented serial MRI sections. This method is fast, operates on anatomic structures of many different sizes, and it allows for comparisons of changes in the location of each individual tendon within the carpal tunnel. Knowledge of individual tendon identity within the carpal tunnel facilitates comparisons of tendon/nerve movement for the same subject performing different activities, and similar comparisons between different subjects performing the same activity. Once known, individual tendon locations and shapes can be related to the median nerve location and shape, and to overall carpal tunnel morphology, thereby providing a wealth of information about the mechanical interactions between these structures, interactions that plausibly contribute to development/progression of CTS. This body of knowledge allows for identifying numerous other functional anatomic measures that may identify reasons for the development of CTS better than more traditional metrics of carpal tunnel anatomy measured from MRI sections (nerve/tunnel size, shape, flattening ratios, etc.). The tendon adjacency measure reported in this work is one example of such a metric that can be developed from knowledge of individual tendon identity. Additionally, this work can be extended to complement other investigative tools such as finite element analysis, which offers a means to provide useful information about mechanical insult to the median nerve. Knowing the exact identity of each tendon within the tunnel allows realistic motions to be prescribed for each structure, which is necessary for driving finite element models. The algorithm's performance speed was such that in the future, large numbers of cases can be expeditiously processed, thereby allowing exploration of pertinent functional questions, such as the potential relationship between median nerve adjacency percentage and CTS prevalence.

There are several limitations to this study. The first is the relatively modest number of subjects that have been evaluated to date using the tendon tracking methodology, and similarly the limited number of hand/finger poses that have been investigated. However, the primary goal of this work was to develop a robust methodology that would allow for rapid identification of the individual tendons in a consistent matter, rather than to definitively identify MRI abnormalities of the tendons within the carpal tunnel. The modest subject pool to date served mainly to provide illustrative data regarding performance of the new methodology in scans performed using a variety of hand poses. As part of the illustrative nature of the data collection, subjects of both genders were used in this work to obtain data for different sized hands, and a single subject with carpal tunnel syndrome was used to verify that the tracking algorithm would function in pathological conditions. A broad variety of image series were obtained in order to view the carpal tunnel in a range of different 
wrist positions. While it would have been ideal to have all subjects perform all hand poses and loading activities, the extended positioning protocol required to obtain these poses would have resulted in intractably long scan times. Additionally, a few of the scans from each individual were omitted from analysis because motion artifact degraded the image quality and the tunnel structures could not be reliably segmented. Again, because the purpose of collecting scans was to provide a wide variety of illustrative data, the best scans encompassing a wide variety of hand postures were analyzed in this work. Thus, the wrist and finger positions of the subjects were not identical, nor were the number of scans for each subject. Future studies using this algorithm to conclusively identify differences between CTS patients and normal individuals should use identical sets of hand/finger positions and loading activities.

Another limitation to this study lies in the evaluation of shape measures made using the axial section plane of the MR sections. Normal carpal tunnel anatomy is conventionally imaged using axial sections taken through the wrist, $2,8,9,23$ but this does not ensure that a given section is necessarily perpendicular to the tendons or to the nerve. Therefore, future work with this methodology should include a transformation to ensure that cross-sectional shape measures that are being compared are true cross sections through the $3 \mathrm{D}$ structure, rather than oblique sections through the structures as they happen to rest in the axial plane of the MRI scanner. Also, the specific choice of $1 \mathrm{~mm}$ proximity as a criterion for constituting adjacency was necessarily a judgment based on our experience with image resolution and segmentation precision. Obviously, other proximity threshold values could equally be implemented.

Despite these limitations, the methodology yielded novel observations regarding geometric relationships within the carpal tunnel. Conventional shape measures (cross-sectional area, perimeter, flattening ratios) arguably proved to be insensitive measures for comparison, since they often showed no consistently discernable trends, despite instances of dramatic shifts of relative nerve/tendon appositions observed in the 3D models. Arrangement, movement, and location hold strong potential as more informative measures. Such information to date has been catalogued only for selected structures, and only in a few observational MRI studies. ${ }^{16,20,24}$ Such studies have found that within the carpal tunnel, the median nerve is usually slightly ovular, and it typically resides superficial (volar) to the digital flexor tendons and deep to the transverse carpal ligament. ${ }^{2,9,25}$ There is slight variation as to where along the transverse carpal ligament the nerve rests when in this location, but most frequently it is located on the radial side of the tunnel. Another common positional variant of the nerve is interposed between the flexor pollicis longus and the superficial tendon of the index finger. ${ }^{2,20,24}$

This tendon "stacking" variability becomes even more important when looking at spatial relationships within the tunnel while the hand and wrist are in different postures, because it has been previously established that there is more tunnel structure movement with changes in hand and wrist position. For example, Zeiss and colleagues investigated the position of tunnel structures with movement of the wrist, showing that in $45^{\circ}$ of extension, the flexor tendons moved to the dorsal portion of the tunnel, leaving the median nerve on the volar side of the tunnel, adjacent to the transverse carpal ligament. ${ }^{24}$ Repositioning of the nerve during $45^{\circ}$ of wrist flexion was more varied, but it typically followed one of three motion paths: remaining adjacent to the transverse carpal ligament, moving between the flexor pollicis longus and the superficial index flexor tendon, or moving between the superficial tendons of the index and long fingers. ${ }^{20,24}$

While these earlier studies provide some information about the general location of the median nerve relative to other structures within the carpal tunnel, they usually omit the specific arrangement of the individual tendons and therefore provide no information about specific tendon/nerve interactions that occur during the movement of the hand and wrist. Those few studies that have included individual tendon information have been limited in the number of structures that could be practically evaluated, owing to the laborious manual tracing heretofore required to definitively identify tendons within the carpal tunnel. The present work shows that like nerve position, tendon arrangement is also highly variable between subjects and between postures. Rapid evaluation of the arrangement of individual tendons relative to the nerve is a prerequisite for evaluation of a large number of specific tendon/nerve interactions associated with hand movement. The specifics of such tendon-nerve interactions could profoundly influence contact stress on the median nerve from adjacent tendons, and thereby contribute to the development of CTS.

In conclusion, we report a new computational methodology that allows for the rapid identification of the individual digital flexor tendons within the carpal tunnel by means of automatically tracking tendon identity from a more distal location in the hand where identity is unambiguous. The region-growing technique utilized operates on MR images of the carpal tunnel, and assigns consistent labeling to each dataset to which it is applied. This allows for comparisons 
of all carpal tunnel structures to be made for a single subject over a range of hand poses, or between a series of different subjects. Conventional shape measures calculated for each positively identified tendon showed inconclusive trends between different subjects and between different hand poses for the same subject, despite instances of dramatic repositioning of the respective carpal tunnel structures. Individual identification of the tendons allowed for quantification of the fraction of the median nerve perimeter that was engaged by specific tendons, and it allowed for quantifying changes in the amount of nerve boundary adjacent to those tendons. Building upon the preliminary data collected, this methodology will allow for measuring potentially CTS-related abnormalities of interaction between the different structures present in the carpal tunnel.

\section{Acknowledgments}

The authors would like to acknowledge the National Institutes of Health for financially supporting this work (AR053899). The authors would also like to acknowledge the work of Mr. Daniel Jaramillo, who assisted with performing the segmentations of the carpal tunnel structures. The authors report no conflicts of interest in this work.

\section{References}

1. Phalen GS. The carpal-tunnel syndrome. Seventeen years' experience in diagnosis and treatment of six hundred fifty-four hands. J Bone Joint Surg Am. 1966;48(2):211-228.

2. Mesgarzadeh M, Schneck CD, Bonakdarpour A. Carpal tunnel: MR imaging. Part I. Normal anatomy. Radiology. 1989;171(3):743-748.

3. Mesgarzadeh M, Schneck CD, Bonakdarpour A, Mitra A, Conaway D. Carpal tunnel: MR imaging. Part II. Carpal tunnel syndrome. Radiology. 1989;171(3):749-754.

4. Allmann KH, Horch R, Uhl M, et al. MR imaging of the carpal tunnel. Eur J Radiol. 1997;25(2):141-145.

5. Buchberger W. Radiologic imaging of the carpal tunnel. Eur J Radiol. 1997;25(2):112-117.

6. Middleton WD, Kneeland JB, Kellman GM, et al. MR imaging of the carpal tunnel: normal anatomy and preliminary findings in the carpal tunnel syndrome. AJR Am J Roentgenol. 1987;148(2):307-316.
7. Helms CA, Major NM, Anderson MW, Kaplan PA, Dussault R. Musculoskeletal MRI. Philadelphia, PA: Elsevier; 2009.

8. Weiss KL, Beltran J, Shamam OM, Stilla RF, Levey M. High-field MR surface-coil imaging of the hand and wrist. Part I. Normal anatomy. Radiology. 1986;160(1):143-146.

9. Girgis WS, Epstein RE. Magnetic resonance imaging of the hand and wrist. Semin Roentgenol. 2000;35(3):286-296.

10. Kleindienst A, Hamm B, Lanksch WR. Carpal tunnel syndrome: staging of median nerve compression by MR imaging. J Magn Reson Imaging. 1998;8(5):1119-1125.

11. Keir PJ. Magnetic resonance imaging as a research tool for biomechanical studies of the wrist. Semin Musculoskelet Radiol. 2001;5(3):241-250.

12. Widgerow AD, Sacks L, Greenberg D, Becker PJ. Intergroup comparisons of carpal tunnel dimensions. J Hand Surg [Am]. 1996;21(3): 357-359.

13. Cobb TK, Bond JR, Cooney WP, Metcalf BJ. Assessment of the ratio of carpal contents to carpal tunnel volume in patients with carpal tunnel syndrome: a preliminary report. J Hand Surg Am. 1997;22(4):635-639.

14. Horch RE, Allmann KH, Laubenberger J, Langer M, Stark GB. Median nerve compression can be detected by magnetic resonance imaging of the carpal tunnel. Neurosurgery. 1997;41(1):76-82.

15. Radack DM, Schweitzer ME, Taras J. Carpal tunnel syndrome: are the MR findings a result of population selection bias? AJR Am J Roentgenol. 1997;169(6):1649-1653.

16. Steinbach LS, Smith DK. MRI of the wrist. Clin Imaging. 2000; 24(5):298-322.

17. Timins ME, O'Connell SE, Erickson SJ, Oneson SR. MR imaging of the wrist: normal findings that may simulate disease. Radiographics. 1996;16(5):987-995.

18. Hobby JL, Dixon AK, Bearcroft PW, et al. MR imaging of the wrist: effect on clinical diagnosis and patient care. Radiology. 2001;220(3):589-593.

19. Keir PJ, Wells RP. Changes in geometry of the finger flexor tendons in the carpal tunnel with wrist posture and tendon load: an MRI study on normal wrists. Clin Biomech (Bristol, Avon). 1999;14(9):635-645.

20. Skie M, Zeiss J, Ebraheim NA, Jackson WT. Carpal tunnel changes and median nerve compression during wrist flexion and extension seen by magnetic resonance imaging. J Hand Surg Am. 1990;15(6):934-939.

21. Mogk JP, Keir PJ. Evaluation of the carpal tunnel based on 3-D reconstruction from MRI. J Biomech. 2007;40(10):2222-2229.

22. Monagle K, Dai G, Chu A, Burnham RS, Snyder RE. Quantitative MR imaging of carpal tunnel syndrome. AJR Am J Roentgenol. 1999;172(6):1581-1586.

23. Yu JS, Habib PA. Normal MR imaging anatomy of the wrist and hand. Radiol Clin North Am. 2006;44(4):569-581.

24. Zeiss J, Skie M, Ebraheim N, Jackson WT. Anatomic relations between the median nerve and flexor tendons in the carpal tunnel: MR evaluation in normal volunteers. AJR Am J Roentgenol. 1989;153(3):533-536.

25. Howe FA, Saunders DE, Filler AG, et al. Magnetic resonance neurography of the median nerve. Br J Radiol. 1994;67(804):1169-1172.
Orthopedic Research and Reviews

\section{Publish your work in this journal}

Orthopedic Research and Reviews is an international, peer-reviewed, open access journal that focusing on the patho-physiology of the musculoskeletal system, trauma, surgery and other corrective interventions to restore mobility and function. Advances in new technologies, materials, techniques and pharmacological agents are particularly welcome. The journal welcomes

\section{Dovepress}

original research, clinical studies, reviews \& evaluations, expert opinion and commentary, case reports and extended reports. The manuscript management system is completely online and includes a very quick and fair peer-review system, which is all easy to use. Visit http://www.dovepress. com/testimonials.php to read real quotes from published authors. 